\title{
NAFLD
}

\section{PNPLA3 VARIANT AND HEPATIC STEATOSIS}

New research provides direct evidence that physiological expression of an lle148Met variant in the endogenous gene encoding patatin-like phospholipid domain containing protein 3 (Pnpla3) causes NAFLD in mice on a high-sucrose diet.

A single nucleotide polymorphism (rs738409, encoding lle148Met) in PNPLA3 in humans is strongly associated with the full spectrum of NAFLD (from simple steatosis to NASH, cirrhosis and hepatocellular carcinoma). Despite numerous studies supporting this link, the underlying mechanisms remain unclear. Existing research had shown that overexpression of the human PNPLA3 Ile148Met variant in mice causes hepatic steatosis. However, Smagris et al. wanted to develop a mouse model that more accurately reflects the physiological phenotype in humans by augmenting the mouse Pnpla3 protein itself.

The researchers introduced a single nucleotide change $(T>G)$ at codon 148 of Pnpla3 in the mouse genome, leading to the lle148Met substitution in the protein (Pnpla3 ${ }^{148 M}$ knockin mice). They also generated a second mouse model in which the mouse genome was altered to introduce a Ser47Ala substitution, resulting in catalytically inactive Pnpla3 (Pnpla3 ${ }^{47 A}$ knockin mice).

Hepatic triglyceride levels in knockin mice on a regular chow diet did not differ from those of wild-type mice; however, both the Pnpla3 ${ }^{148 M}$ and Pnpla $3^{47 A}$ knockin mice developed hepatic steatosis (2-3-fold increase in hepatic fat) on a high-sucrose diet. These increased levels of liver fat were accompanied by a notable 40 -fold increase in variant Pnpla3 on hepatic lipid droplets, but no increase in hepatic Pnpla3 mRNA or major alterations in fatty acid composition of major hepatic lipids.

"These findings demonstrate that the lle148Met substitution is not equivalent to loss of protein expression (as illustrated by the lack of steatosis in Pnpla3knockout mice)," say authors Helen Hobbs and Jonathan Cohen, "rather, Pnpla3associated hepatic steatosis in mice requires the presence of the catalytically inactive protein." More work is needed to further clarify the relationship between PNPLA3 accumulation, lipid droplet size and hepatic steatosis, as well as the association between PNPLA3 and diet.

Katrina Ray

Original article Smagris, E. et al. Pnpla31148M knockin mice accumulate PNPLA3 on lipid droplets and develop hepatic steatosis. Hepatology doi:10.1002/hep.27242 